\title{
Vocal cord paralysis due to self-expandable metal stent in the proximal esophagus
}

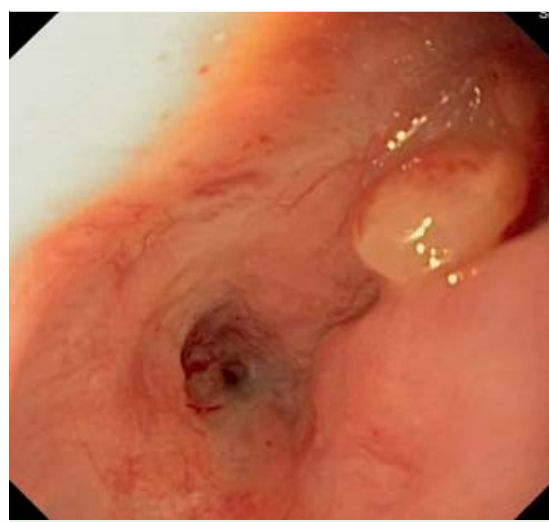

Fig. 1 Endoscopic view of the anastomotic pinpoint stenosis with a proximal lateral fistula immediately distal to the upper esophageal sphincter.

A 56-year-old man was referred for endoscopic treatment of benign postoperative stenosis of an esophagogastric anastomosis with a proximal fistula to the neck. He had previously undergone chemo- and radiotherapy for squamous cell carcinoma of the proximal esophagus. Because of recurrent malignancy, esophageal resection with tubular gastric reconstruction was performed. Postoperative dysphagia and cutaneous fistula to the neck occurred. Endoscopic examination revealed pinpoint stenosis at $2 \mathrm{~cm}$ distal to the upper esophageal sphincter with a fistula below the sphincter $(\bullet$ Fig. 1 ).

Under fluoroscopic guidance, and with the patient under general anesthesia, balloon dilation of the stenosis up to $8 \mathrm{~mm}$ was performed and an 8-cm-long, fully covered self-expandable metal stent (SEMS) was placed, covering the fistula ( Fig.2). Barium swallow confirmed sealing of the fistula and good stent position and deployment ( $\mathbf{F i g}$.3). The patient experienced no pain or dysphagia and was allowed to eat semifluids.

Four days later he was admitted to the emergency room because of hoarseness and severe stridor. Endoscopy showed bilateral vocal cord paralysis without edema or compression and a good stent position immediately below the upper esophageal sphincter ( Fig.4). After endotracheal intubation, the stent was removed by inversion, revealing a dilated stenosis ( $\bullet$ Fig.5). After weaning 2 days later, the vocal cord paralysis gradually improved

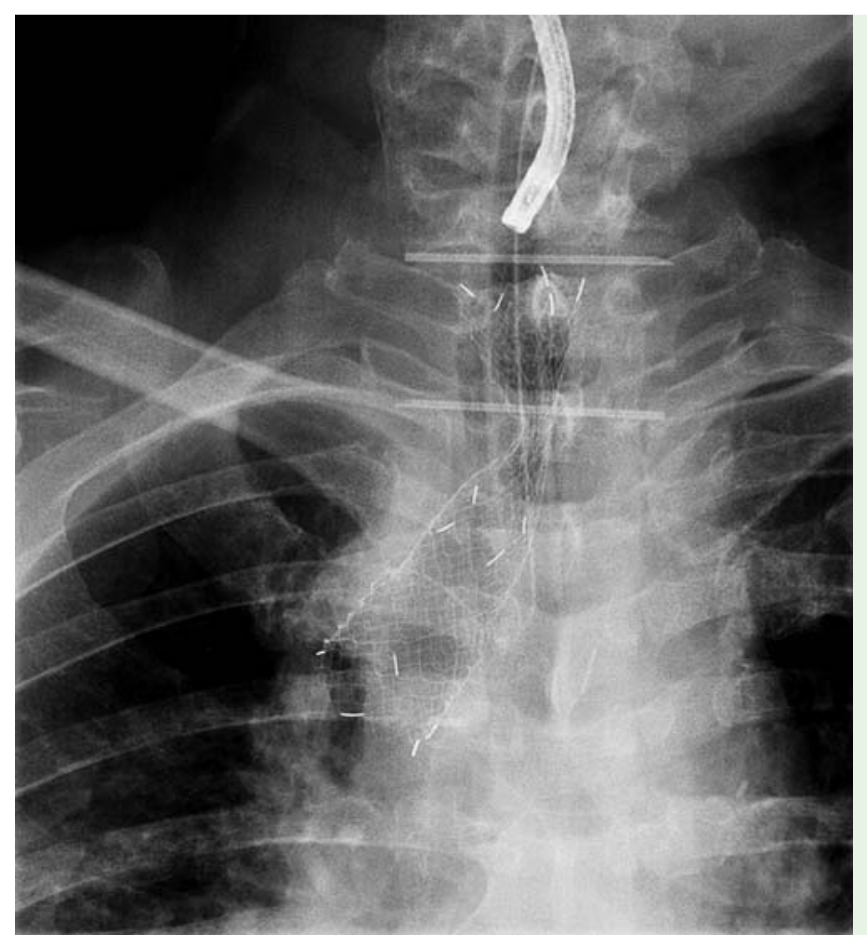

Fig. 2 Placement of a fully covered metal stent under fluoroscopic guidance with two external markers identifying the location of the upper esophageal sphincter and the stenosis.

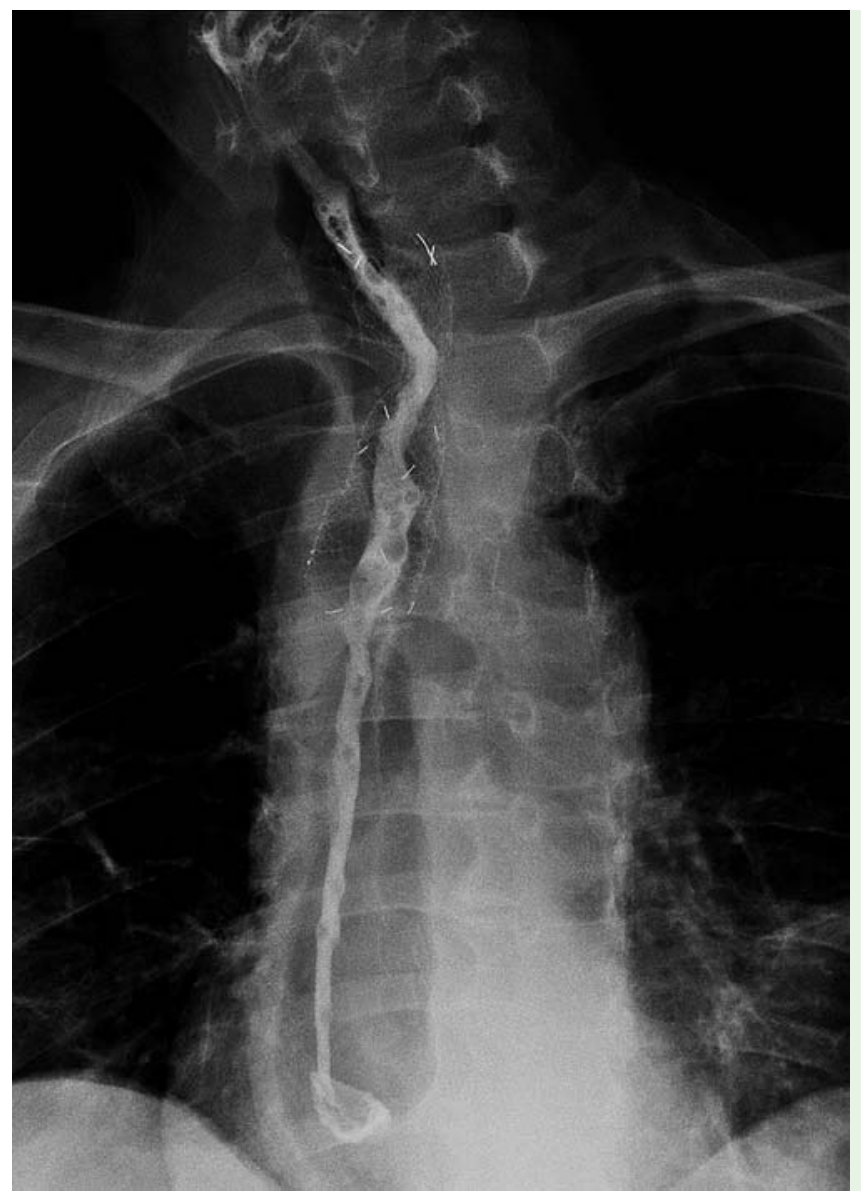

Fig. 3 Barium swallow confirming closure of the internal opening of the proximal cutaneous fistula. 


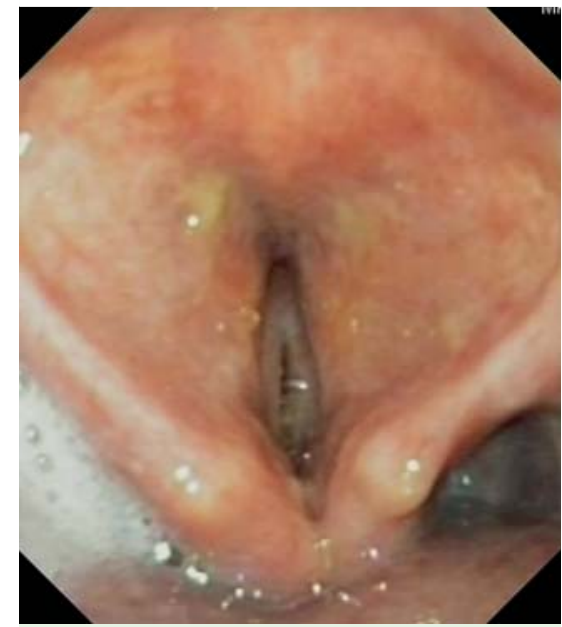

Fig. 4 Endoscopic view of the bilateral vocal cord paralysis 4 days after placement of a fully covered metal stent resulting in severe stridor.

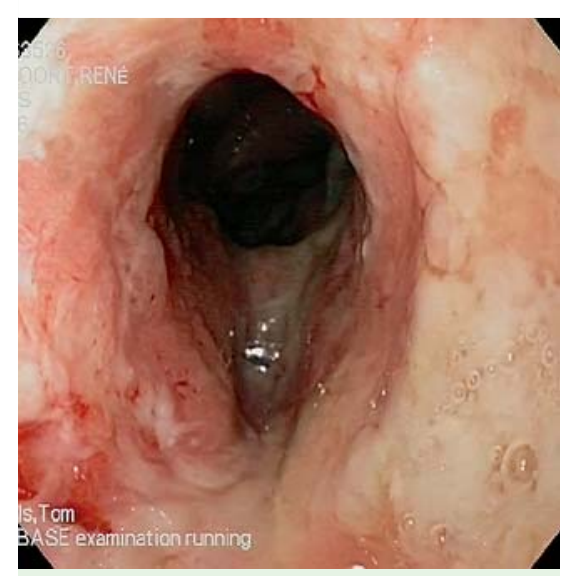

Fig. 5 Endoscopic view of the stenosis after stent removal.

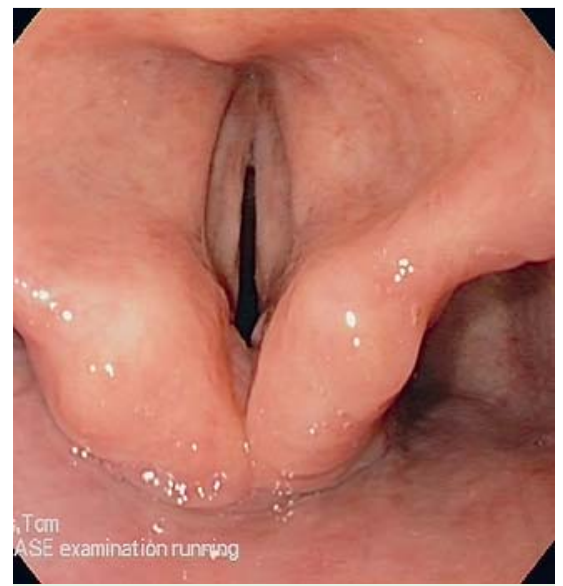

Fig. 6 Endoscopic view of gradual recovery of bilateral vocal cord paralysis.

and spontaneous respiration without stridor was possible ( $\bullet$ Fig. 6 ).

This case illustrates the risk of SEMS placement in the proximal esophagus [1]. Progressive hoarseness and stridor occurred, but improved gradually after removal of the stent. This is in contrast to a previous report in which a tracheostomy was required because of permanent vocal cord paralysis [2]. Since no compression or edema occurred in our patient, it is hypothesized that the proximal flare of the stent compressed the recurrent laryngeal nerve, resulting in vocal cord paralysis [3]. Fortunately, the patient gradually recovered after the stent was removed. Transient vocal cord paralysis is considered a rare complication of SEMS placement in the proximal esophagus.

Endoscopy_UCTN_Code_CPL_1AH_2AD

Competing interests: None
Tom G. Moreels ${ }^{1}$, Heiko U.

De Schepper ${ }^{1}$, Elisabeth J. Macken ${ }^{1}$, Guy J. Hubens ${ }^{2}$, Paul A. Pelckmans ${ }^{1}$

${ }^{1}$ Department of Gastroenterology and Hepatology, Antwerp University Hospital, Antwerp, Belgium

2 Department of Abdominal Surgery, Antwerp University Hospital, Antwerp, Belgium

\section{References}

1 Verschuur EML, Kuipers EJ, Siersema PD. Esophageal stents for malignant strictures close to the upper esophageal sphincter. Gastrointest Endosc 2007; 66: 1082 - 1190

2 Gellad ZF, Hampton D, Tebbit CL et al. Bilateral vocal cord paralysis following stent placement for proximal esophageal stricture. Endoscopy 2008; 40 (Suppl. 02): E150

3 Vachha B, Cunnane MB, Mallur $P$ et al. Losing your voice: etiologies and imaging features of vocal cord paralysis. J Clin Imaging Sci 2013; 3: 15. doi: 10.4103/ 2156-7514.109751

\section{Bibliography}

DOI http://dx.doi.org/

10.1055/s-0034-1364954

Endoscopy 2014; 46: E155-E156

(c) Georg Thieme Verlag KG

Stuttgart · New York

ISSN 0013-726X

\section{Corresponding author}

\section{Tom G. Moreels, MD, PhD}

Department of Gastroenterology and Hepatology Antwerp University Hospital

Wilrijkstraat 10

2650 Edegem-Antwerp

Belgium

tom.moreels@uza.be 